\title{
The (sub-)milliarcsecond scale structure of the nucleus of the Sombrero galaxy
}

\author{
Kazuhiro Hada*1, Akihiro Doi ${ }^{2}$, Hiroshi Nagai ${ }^{3}$, Makoto Inoue ${ }^{4}$ \\ ${ }^{1}$ INAF Istituto di Radioastronomia, Via Gobetti 101, 40129 Bologna, Italy \\ ${ }^{2}$ Institute of Space and Astronautical Science, 3-1-1 Yoshinodai, Sagamihara, 229-8510 \\ Kanagawa, Japan \\ ${ }^{3}$ National Astronomical Observatory of Japan, 2-21-1 Osawa, Mitaka, 181-8588 Tokyo, Japan \\ ${ }^{4}$ Academia Sinica Institute for Astronomy and Astrophysics, $11 \mathrm{~F}$ Astronomy-Mathematics Bldg., \\ National Taiwan University, No. 1, Roosevelt Rd., Sec. 4 Taipei 10617, Taiwan R.O.C. \\ E-mail: hada@ira.inaf.it
}

\begin{abstract}
Sombrero galaxy (M 104, NGC 4594) is one of the nearest low-luminosity active galactic nuclei. This source provides an excellent case to study the accretion and ejection processes in the vicinity of extremely sub-Eddington black holes due to its proximity and large black hole mass. Here we present preliminary results of our deep multi-frequency VLBA observation of the Sombrero nucleus. We detected the nucleus at the seven frequencies between 1.4 and $43 \mathrm{GHz}$, and successfully obtained high-resolution images on a scale less than 200 Schwarzschild radii. Moreover, we discovered a two-sided structure extending from the radio core, which can be interpreted as the nuclear radio jets.
\end{abstract}

11th European VLBI Network Symposium \& Users Meeting

9-12 October 2012

Bordeaux (France)

\footnotetext{
* Speaker.
} 


\section{Introduction}

M 104 (NGC 4594) is a famous early-type spiral galaxy well-known as the "Sombrero galaxy". It is located in the southern hemisphere at a distance of $D=9.0 \pm 0.1 \mathrm{Mpc}$ [1] and shows a nearly edge-on view ( $84^{\circ}$ from our line-of-sight). Hubble Space Telescope observations indicates the presense of a supermassive black hole weighing $1 \times 10^{9} M_{\odot}$ in the center [2, 3]. The nucleus of this source shows a highly sub-Eddington luminosity $\left(L_{\mathrm{bol}} \sim 2.5 \times 10^{41} \mathrm{erg} \mathrm{s}^{-1} \sim 2 \times 10^{-6} L_{\text {Edd }}\right.$, [4]) with the absense of the big blue bump in its spectral energy distribution (SED) [5], making this source into a prototypical class of low-luminosity active galactic nuclei (LLAGN).

Detailed physical processes acting in the vicinity of the LLAGN central engines remain as a major question in the local Universe (e.g., [6] and references therein). To study this issue, the Sombrero provides a particularly suitable opportunity because of its closeness and a large estimated black hole mass, the combination of which yields 1 milliarcsecond $=0.04 \mathrm{pc}=454 R_{\mathrm{s}}$. This physical scale in $R_{\mathrm{S}}$ unit is more than five times closer to the black hole than that of another wellinvestigated LLAGN M 81, or even comparable to the scales obtained for SgrA* or M 87.

In spite of such an observational advantage, however, very little is known about its milliarcsecond scale structure because of the quite limited number of VLBI observations so far. Early VLBI experiments of this nucleus indicate a simple compact structure without any signs of extended emission ([7], [8], [9]). Here we report on some preliminary results on our new, dedicated VLBI observations toward the Sombrero nucleus, which are much deeper than any other previous VLBI experiments. Thanks to the capabilities of quasi-simultaneous, multi-frequency, phase-referencing mode, the observations bring about lots of new findings on the milliarcsecond-scale properties of this source.

\section{Observations and Data Reduction}

We observed M 104 on March 23 and 302011 with VLBA at 2.3, 5.0, 8.4, 15.4, 23.8 and 43.2 GHz quasi-simultaneously. The observations involved fast switching between M 104 and a nearby bright radio source J1239-1023, which is separated by 1.23 on the sky, plus a few calibrators. Each observation was conducted in a 10-hour overnight run at a data recording speed of $512 \mathrm{Mbps}$. The initial data calibration was performed using the NRAO AIPS based on the standard VLBI data reduction procedures. The amplitude calibration with the opacity corrections was applied using the measured system noise temperature and the elevation-gain curve of each antenna. To remove residual delays, rates and phases, we first tried fringe-fitting on each source separately. We clearly detected the fringes with sufficient signal-to-noise ratios (SNR), except for the M 104 data at $43 \mathrm{GHz}$. At this frequency, we then transferred the solutions derived by J1239-1023 to the time segment of M 104 (phase-referencing technique). Because J1239-1023 has a complicated structure, we corrected the deviation of the phase/gain solutions from a point source model by using a self-calibrated image of this source. Through this process, we successfully recovered the fringes of M 104 at $43 \mathrm{GHz}$. Images were created in DIFMAP software with iterative phase/amplitude self-calibration. The amplitude self-calibration for M 104 was performed with a relatively long solution interval ( $60 \sim 240$ minutes, depending on SNR of each data). 

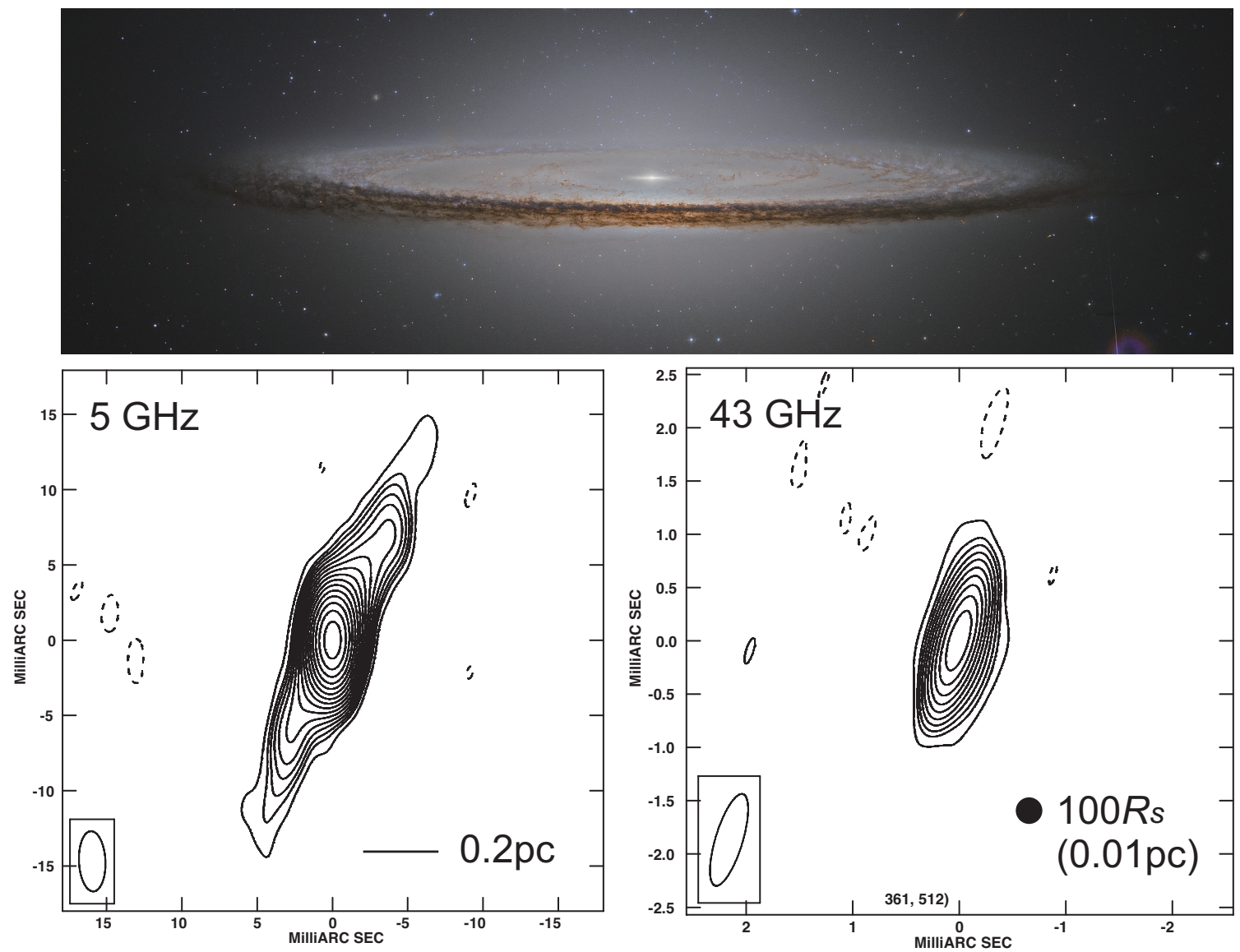

Figure 1: (Top) Optical image of the Sombrero (Credit \& Copyright: Vicent Peris (OAUV / PTeam), MAST, STScI, AURA, NASA). (Bottom) VLBA images of the Sombrero nucleus at 5 and $43 \mathrm{GHz}$. The contour starts from $-1,1,2$ times of $3 \sigma$ image rms and thereafter increasing by factor of $\sqrt{2}$. The peak fluxes are 67 and $73 \mathrm{mJy} / \mathrm{beam}$ for 5 and $43 \mathrm{GHz}$ images, respectively.

Also, we found one VLBA archival data of M 104 at $1.4 \mathrm{GHz}$, which was observed in April 2008 with a relatively long integration time. Although the epoch is separated by $\sim 3$ years from our observations, this data is still quite helpful to understand the nuclear structure at lower frequencies. We then include this data in our study.

\section{Preliminary Results and Discussion}

In Figure 1 we show self-calibrated images of M 104 at 5 and $43 \mathrm{GHz}$. Thanks to highly improved sensitivities, we successfully produced the best quality VLBI images ever obtained for this source. To our knowledge, this is the first clear VLBI detection and imaging at higher than $15 \mathrm{GHz}$. In particular at $43 \mathrm{GHz}$, where the angular resolution is the highest, we have resolved the structure down to $\lesssim 200 R_{\mathrm{S}}$ scale. After the nearby famous targets of SgrA*, M 87 and Centaurus A, M 104 becomes the fourth source in which the nuclear structure was resolved on such a close vicinity of the central black hole. 

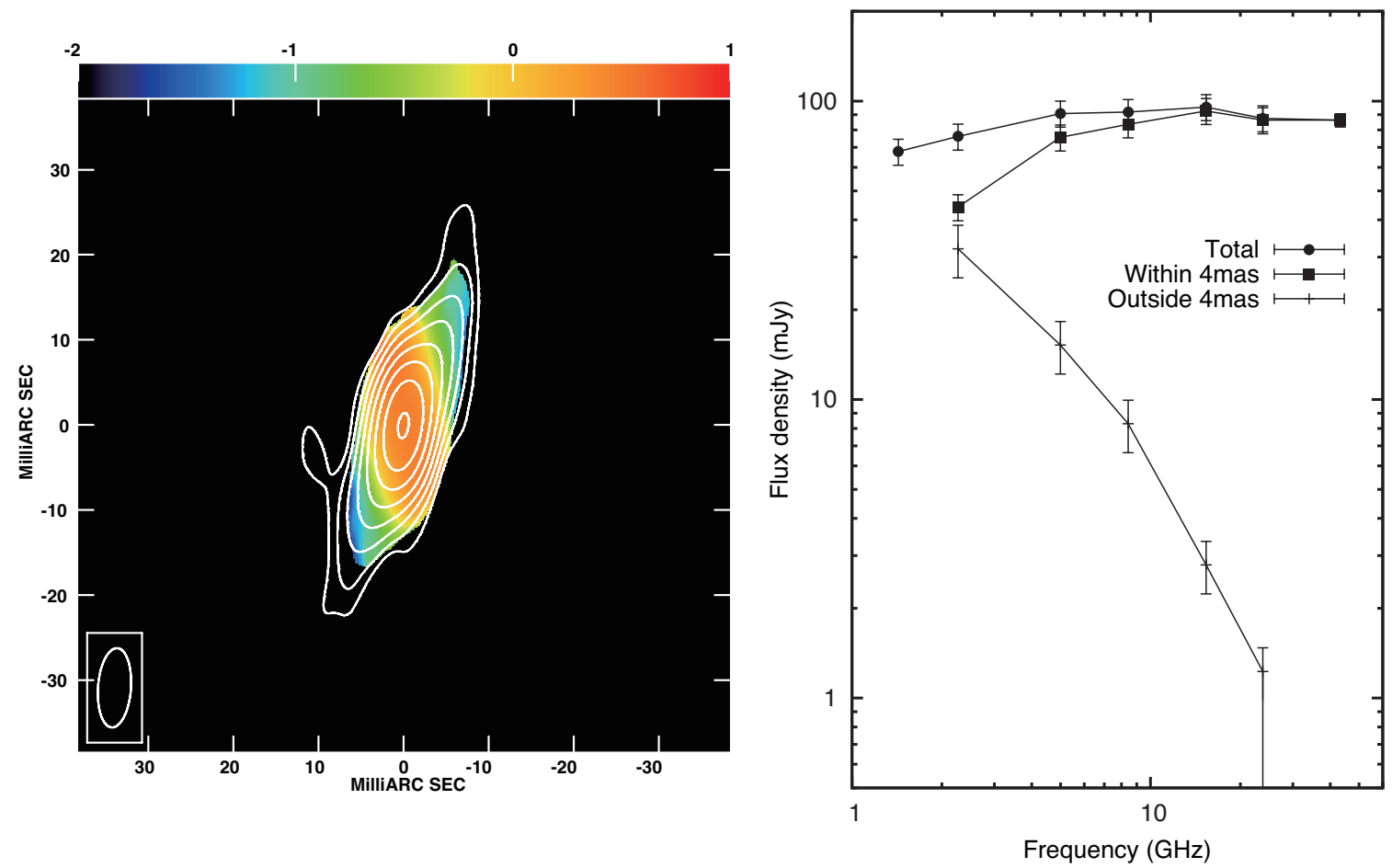

Figure 2: (Left) Spectral index distribution map of M 104 between 2 and 5 GHz (colours). Two images are convolved with a common 2-GHz beam and aligned with each phase center. Contours indicate an intensity distribution at $2 \mathrm{GHz}$, staring from $3 \sigma \mathrm{rms}$ level. (Right) Observed VLBI spectra between 1.4 and $43 \mathrm{GHz}$. Three different regions are shown: (1) entire region on VLBI scale, (2) central region (within 4-mas-diameter circle centered on the brightness peak, corresponding to $\sim 2 \times 10^{3} R_{\mathrm{S}}$ ), (3) outer region (outside the 4-mas circle).

At lower frequencies, we have clearly detected an extended structure which elongates from the core. This is the first discovery of such emission on the mas-scale structure. This structure seems to be smooth with a symmetric shape about a north-south direction, and extends the entire length of $\sim 120$ mas and $\sim 30$ mas at 1.4 and $5 \mathrm{GHz}$ (above $3 \sigma$ level), respectively. Compared with this elongation, the structure in the east-west direction is narrow. As frequency increases, the structure becomes gradually compact, and the radio core increasingly dominates the entire radio emission. At $43 \mathrm{GHz}$, most of the emission is confined within 1 mas. A tentative Gaussian modelfitting to the $43-\mathrm{GHz}$ data yields a size of the radio core as $\lesssim 0.25$ mas $\left(0.01\right.$ pc or $\left.113 R_{\mathrm{S}}\right)$ with a flux density of $\sim 85 \mathrm{mJy}$. The brightness temperature of the core is estimated to be around $\gtrsim 2 \times 10^{9} \mathrm{~K}$.

Multi-frequency images of M 104 indicates the nuclear structure to be frequency dependent. In the left-hand side of Figure 2 we show a spectral index map between 2 and $5 \mathrm{GHz}$ (here we define spectral index $\alpha$ as $S_{v} \propto v^{+\alpha}$ where $S_{v}$ denotes flux density at a frequency $v$ ). The spatial gradient of the spectral index distribution is apparent along the elongation. The spectrum is slightly inverted near the core and progressively become steep as receding from the core towards both north/south directions. The detailed spectral properties become much clearer by combining all of the multi-frequency data. The right-hand side of Figure 2 shows broadband spectra between 1.4 and $43 \mathrm{GHz}$ for different regions. Over this frequency coverage, the central part (defined as 
the region within 4-mas-diameter circle centered on the core) shows an inverted spectrum with an averaged spectral index of $\alpha \sim 0.18$, whereas the outer part (outside the 4-mas circle) indicates a steep spectrum $(\alpha \sim-1.3)$. The sum of these parts produces an integrated spectrum with $\alpha \sim 0.05$, which is slightly flatter than a non-simultaneous spectrum previously obtained on the arcsecondscale nucleus ([10], [11]). Note that similar spatial gradients of $\alpha$ are seen also in the nucleus of M 81 ([12], [13]).

The observed high brightness temperature, two-sided symmetric structure and spectral characteristics of this nucleus are reminiscent of those of higher luminosity AGN such as FR I or FR II type radio galaxies, in which most of the radio emission is dominated by nonthermal synchrotron emission from relativistic jets with varying nuclear opacity. Considering these similarities, the observed nuclear radio structure of M 104 can be interpreted as a downsized version of nonthermal jets. The presence of nuclear radio jets in M 104 is indeed consistent with a theoretical expectation based on modeling of the broadband SED [14], and also with the nuclear properties at X-ray [4]. Further information derived from astrometric core shift measurements [15] will also be helpful to understand the detailed properties of this structure.

Acknowledgments. The Very Long Baseline Array is operated by the National Radio Astronomy Observatory, a facility of the National Science Foundation, operated under cooperative agreement by Associated Universities, Inc. KH is supported by the Canon Foundation in Europe.

\section{References}

[1] Spitler, L. R., Larsen, S. S., Strader, J., et al. 2006, AJ, 132, 1593

[2] Kormendy, J. 1988, ApJ, 335, 40

[3] Kormendy, J., et al. 1996, ApJ, 473, L91

[4] Pellegrini, S., Baldi, A., Fabbiano, G., \& Kim, D.-W. 2003, ApJ, 597, 175

[5] Ho, L. 1999, ApJ, 516, 672

[6] Ho, L. C. 2008, ARA\&A, 46, 475

[7] Shaffer, D. B., \& Marscher, A. P. 1979, ApJ, 233, L105

[8] Graham, D. A., Weiler, \& K. W.,Wielebinski, R. 1981, A\&A, 97, 388

[9] Preston, R. A., et al. 1985, AJ, 90, 1599

[10] de Bruyn, A. G., Crane, P. C., Price, R. M., \& Carlson, J. B. 1976, A\&A, 46, 243

[11] Hummel, E., van der Hulst, J. M., \& Dickey J. M. 1984, A\&A, 134, 207

[12] Bietenholz, M. F., Bartel, N., \& Rupen, M. P. 2004, ApJ, 615, 173

[13] Martí-Vidal, I., Marcaide, J. M., Alberdi, A., et al. 2010, A\&A, 533, A111

[14] Yuan, F., Yu, Z., \& Ho, L. C. 2009, ApJ, 703, 1034

[15] Hada, K., Doi, A., Kino, M., et al. 2011, Nature, 477, 185 